\title{
Conserved Gravitational Charges, Locality and the Holographic Weyl Anomaly - a Fresh Viewpoint
}

\author{
Pedro Lauridsen Ribeiro \\ Departamento de Física Matemática - Instituto de Física, \\ Universidade de São Paulo, CP 66.318, 05315-970, São Paulo, SP, Brazil
}

(Received on 17 October, 2005)

\begin{abstract}
Since the proposal of the AdS/CFT correspondence, made by Maldacena and Witten, there has been some controversy about the definition of conserved Noether charges associated with asymptotic isometries in asymptotically AdS spacetimes, namely, whether they form an anomalous (i.e., a nontrivial central extension) representation of the Lie algebra of the conformal group in odd bulk dimensions or not. In the present work, we shall review the derivation of these charges by using covariant phase space techniques, emphasizing the principle of locality underlying it. We shall also comment on how these issues manifest themselves in the quantum setting.
\end{abstract}

\section{INTRODUCTION}

The issue of defining global conserved charges in General Relativity is quite a delicate one. The difficulties one finds stem from the fact that the dynamical problem posed by Einstein's equations is a rather sui generis one: It does not possess a local dynamics in the usual sense of a "time evolution", because the very choice of "time" is a local (gauge) symmetry of the system (Hamiltonian constraint), and thus the Noether current corresponding to it, which was supposed to generate the dynamics according to the usual Hamiltonian recipe, actually vanishes "on shell". This stems from the deep physical fact, put forward by Mach and Einstein, that one needs a material, physical procedure to fix a local notion of time (namely, to fix a coordinate system), in a way that the physical laws ruling these procedures are also local and moreover independent of this choice - namely, one needs a nonvanishing matter energy-momentum tensor. This is the physical content of Einstein's equations. Another, distinct way of fixing the dynamical interpretation (namely, the improvement terms to be added to the constraints of the theory) is to assume that the metric approaches some fixed, symmetrical background near infinity for which we do know how to fix such an interpretation.

However, one must be careful with formal computations regarding the on-shell action - its value for the whole spacetime may be infinite. This divergence inserts a new ambiguity in the definition of conserved gravitational charges, this one depending only on the conformal structure of infinity. Whereas the usual definition gives a family of charges whose Poisson algebra is a true representation of the algebra of asymptotic isometries, the potential ambiguity stemming from the above divergence may lead to an obstruction to forming a representation[1]. The inception of MaldacenaWitten AdS-CFT correspondence[2, 3], according to which the asymptotic boundary behaviour of bulk fields corresponds to sources for "dual", boundary conformal fields, has led on its turn to another, QFT-inspired prescription, according to which nontrivial (with respect to pure AdS) boundary behaviour of the bulk metric acts as a source for the dual energy-momentum tensor, whose renormalization yields a trace anomaly in even boundary dimensions.

Here, we start from the fact that the variational principle determining dynamics is inherently local. Namely, one must keep track of the supports of the functionals - conserved gravitational charges are quasi-local quantities, and they must be obtained as suitable limits of local quantities. In this sense, conserved gravitational currents are indeed localized at infinity, since local gravitational charges, as we've seen, don't actually exist (more precisely, they vanish everywhere). The near-boundary behaviour ends up being affected by the same kind of ambiguity one faces in the renormalization of QFT[4]. The result is, however, thatthe anomalous terms are actually independent of the detailed behaviour of the bulk metric (they depend only on the conformal class of the boundary metric[5]), and must be fixed by additional, physical considerations. The AdS-CFT recipe[1] is one possible answer.

We review the calculations leading to the above ambiguity in Section 2 in an unified way by using the covariant phase space formalism (Peierls bracket[5]). In Section 3, we propose what we believe to be a QFT counterpart of the action of gravitational charges, based on first principles - namely, locality, covariance and causality. By employing a functorial framework in the spirit of [6], we give a prescription, inspired by our previous classical calculations, which seem to capture their essential aspects.

\section{COVARIANT PHASE SPACE APPROACH TO CONSERVED CHARGES}

Let $\mathscr{M}$ be a $n$-dimensional manifold, $K \Subset \mathscr{M}$ with regular boundary $\partial K$. Define

$$
S_{K}[g, \Lambda]={ }_{K}^{\mathrm{Z}}(R(g)+2 \Lambda) \sqrt{-g} d^{n} x,
$$

(Ric $(g)$ and $R(g)$ are resp. the Ricci tensor and the scalar curvature associated with $g$ ) the Einstein-Hilbert action with cosmological constant $\Lambda$. For variations $\delta g$ supported in the interior of $K$, we have 


$$
\delta S_{K}[g, \Lambda]={ }_{K}^{\mathrm{Z}} \underbrace{\left(\operatorname{Ric}(g)-\frac{1}{2} R(g) g+\Lambda g\right)}_{\doteq G_{a b}^{\Lambda}} \delta g \sqrt{-g} d^{n} x=0
$$

at a $g$ satisfying the Eisntein equations $\operatorname{Ric}(g)-1 / 2 R g+$ $\Lambda g=0$. Now, consider an arbitrary metric (i.e., not necessarily "on shell"), and perform metric variations $\delta g^{a b}=$ $1 / 2 \nabla^{(a} X^{b)}$ coming from (infinitesimal) spacetime diffeomorphisms $X^{a}$. The Bianchi identities imply that:

$$
G_{a b}^{\Lambda} \nabla^{a} X^{b}=\nabla^{b}\left(G_{a b}^{\Lambda} X^{a}\right) .
$$

If $X$ if timelike, one can see that $\mathscr{C}_{a}(g, X) \doteq G_{a b}^{\Lambda} X^{b}$ does not contain second derivatives of the metric in the $X$ direction. In other words, the $\mathscr{C}_{a}(g, X)$ 's are constraints of the theory, expressing its diffeomorphism invariance. Likewise, these constraints determine the dynamics of any gauge-invariant quantity - instead of showing it in general, we'll specialize to asymptotically AdS (AAdS) spacetimes[7], for which the "boundary improvement" procedure mentioned in the Introduction can be implemented rather explicitly.

Let $(\widehat{\mathscr{M}}, \widehat{g})$ be a $n$-dimensional $(n \geq 4)$, AAdS sapcetime with conformal factor $\Omega$, conformal completion $\left(\mathscr{M}, g \doteq \Omega^{2} \widehat{g}\right)$ and conformal boundary $(\mathscr{I} \doteq \partial \mathscr{M}, q \doteq g\lceil\mathscr{I})$, with $q$ lying within the conformal class $[q]$ of the Einstein static universe (ESU). We'll assume that our AAdS spacetimes satisfy empty space Einstein equations everywhere and are causally simple (i.e., the causal past and future of any compact set are closed), hence stably causal, as in [8]. This means they can be foliated by equal-time surfaces (not necessarily Cauchy) by means of a global time function, say $\tau$. We can suppose that $\tau$ can be smoothly extended to $\mathscr{M}$ in such a way that $\tau\lceil\mathscr{I}$ is also a global time function.

We shall consider the following setting: Let $t_{1}<t_{2} \in \mathbb{R}$, $\varepsilon \in[0, \zeta), \zeta$ such that $d \Omega$ vanishes nowhere in the collar $\mathscr{I} \times$ $[0, \zeta)$, and set $\Sigma_{t}=\tau^{-1}(t), \Sigma_{t}^{\varepsilon}=\Sigma_{t} \backslash \Omega^{-1}([0, \varepsilon]$. The ingoing and outgoing null hypersurfaces emanating from $\partial \Sigma_{t_{i}}^{\varepsilon}, i=1,2$ cross resp. in $\Delta_{i}, \Delta_{o}$, forming the edges of the Cauchy surfaces resp. for the regions $\mathscr{O}, \mathscr{O}_{1}$. By choosing $t_{1}$ and $t_{2}$ sufficiently close to each other, we can assure that $\Delta_{i}$ and $\Delta_{o}$ are smooth (in such a case, $\mathscr{O}$ and $\mathscr{O}_{1}$ are regular diamonds) and belong to the same Cauchy surface for $\mathscr{O}_{1}$. We also set $\Omega\left(\Delta_{o}\right) \subset\left[\varepsilon^{\prime}, \varepsilon^{\prime \prime}\right]$, $0<\varepsilon^{\prime}<\varepsilon^{\prime \prime}<\varepsilon$. Finally, we remark that we can deform the orbits of $\tau$ by suitably redefining the latter, in a way that each orbit of $\Sigma_{t} \backslash \Sigma_{t}^{\varepsilon}$ under $\tau$ belong to some level surface of the collar above.

We'll compute all quantities we need from the EinsteinHilbert action $S_{K_{t_{1}, t_{2}, \varepsilon},}[\widehat{g}, \Lambda]$, where $K_{t_{1}, t_{2}, \varepsilon}=\cup_{t \in\left[t_{1}, t_{2}\right]} \Sigma_{t}^{\varepsilon} \Subset \widehat{\mathscr{M}}$. Given an arbitrary vector field $X^{a}$, the variation of the action under $X^{a}\left(\delta \widehat{g}^{a b}=1 / 2 \nabla^{(a} X^{b)}\right)$ reduces to (we'll omit the volume elements for simplicity)

$$
\begin{gathered}
\delta_{X} S_{K_{t_{1}, t_{2},},}[\widehat{g}, \Lambda]=-{ }_{t_{1}}{ }^{\mathrm{z}_{t_{2}} \mathrm{z}} \partial \Sigma_{t}^{\varepsilon}\left(G_{a b}^{\Lambda} \omega^{a} X^{b}+\right. \\
\left.+\omega^{a} \theta\left(\widehat{g}, \mathfrak{L}_{X} \widehat{g}\right)_{a}\right)+{ }_{\Sigma_{l_{2}}^{\varepsilon}-\Sigma_{t_{1}}^{\varepsilon}}^{\mathrm{z}}\left(G_{a b}^{\Lambda} \sigma^{a} X^{b}+\sigma^{a} \theta\left(\widehat{g}, \mathfrak{L}_{X} \widehat{g}\right)_{a}\right)
\end{gathered}
$$

$\left(\omega^{a}\right.$ and $\sigma^{a}$ are the unit ingoing spacelike, resp. future directed timelike normals to the timelike, resp. spacelike smooth piecewise components of $\left.\partial K_{t_{1}, t_{2}, \varepsilon}\right)$. The 1 -form $\theta(\widehat{g}, \delta \widehat{g})$ is Poincare dual (with respect to the volume element $\sqrt{-\widehat{g}}$ ) to the boundary term coming from an arbitrary first variation $\delta \widehat{g}$. Consider now the following, arbitrary second variation, imposing the following conditions on $X^{a}$ :

- $X^{a}$ vanishes in a neighborhood $N_{1}$ of $\Sigma_{t_{1}}$;

- On a neighborhood $N_{2}$ of $\Sigma_{t_{2}}$, disjoint from $N_{1}, X^{a}$ is an asymptotic Killing field, i.e., $\mathfrak{L}_{X}(\widehat{g})$ decays at least as fast as $\varepsilon^{n-2}$ as $\varepsilon \rightarrow 0$ (this implies that the integral of the Lie derivative over $\partial \Sigma_{t}^{\varepsilon}$ has a finite limit as $\varepsilon \rightarrow 0$ ).

This can be done by multiplying an arbitrary asymptotic Killing field $X^{a}$ by a regularized step function. Now here comes the main point: if $\delta \widehat{g}$ is tangent to a curve of solutions of the Einstein equations, it's propagated (in a particular gauge for the linearized equations) by convolution with the covariant derivative along the orbits of $\tau$ of some fundamental solution $E_{c d e f}$ associated with the globally hyperbolic region $\mathscr{O}_{1}$, along the timelike component of the boundary (Duhamel's principle). Let us drop the variation of $\theta$ for now; we shall now exploit the presence of the step function and choose the $r e$ tarded fundamental solution. Putting it all together from (4), we get:

$$
-\delta{ }_{t_{1}}^{{ }_{t_{2}} \mathrm{z}} \partial \Sigma_{t}^{\varepsilon} G_{a b}^{\Lambda} \omega^{a} X^{b}={ }_{t_{1}}^{\mathrm{z}_{t_{2}} \mathrm{z}} \partial \Sigma_{t}^{\varepsilon} \omega^{a} X^{b} \partial_{t} \delta \bar{g}^{a b} .
$$

The calculation of the variation of $\theta$ is more cumbersome and we'll skip it due to lack of space[5]. Antisymmetrization of the second variation and repetition of the procedure above leads to the usual expression for the charges, which is no longer dependent neither on the regularized step function, nor on $t$ :

$$
Q(X) \propto \lim _{\varepsilon \rightarrow 0}^{\mathrm{z}}{ }_{\partial \Sigma_{t}^{\varepsilon}} \Omega^{3-n} C_{a b c d}(\widehat{g}) \nabla^{a} \Omega \nabla^{b} \Omega X^{c} \sigma^{d},
$$

where $C_{a b c d}(\widehat{g})=C_{a b c d}(g)$ is the Weyl tensor of $\widehat{g}$. Notice that the limit is taken by simultaneously taking $t_{1} \rightarrow t_{2}$ and shrinking the regularized step function to the Heaviside step function, so that $\mathscr{O}_{1}$ remains globally hyperbolic all the way - otherwise, the retarded fundamental solution may cease to exist or no longer be unique. The requirement that the variation of the charges should act as boundary sources for linearized gravity around a solution of Einstein's equations fix the charges themselves up to dynamically trivial terms. 
More precisely, it fixes their Peierls bracket with compactly supported, gauge invariant functionals of the on shell metric (local observables). The remaining ambiguity has vanishing Peierls bracket with all local observables and depends on the scaling properties of the local charges w.r.t. $\Omega$ as the limit is taken, as it arises from the ill-defined multiplication of the limit retarded fundamental solution by a Heaviside step function. Depending on the asymptotic scaling degree[4], the needed extension to $\mathscr{I}$ defined by fixing the ambiguity may acquire unavoidable logarithmic terms, violating the expected scaling behaviour. This happens, for instance, for $n$ odd[1].

\section{THE PICTURE FROM LOCAL QUANTUM PHYSICS}

The conceptual advantage of employing the Peierls bracket in the classical calculations above is that it brings the principle of locality to the forefront, in a way akin to QFT. One can emulate the line of reasoning above within local quantum physics (algebraic QFT[9]) by means of the functorial formalism proposed in [6]. Let $\underline{\mathscr{M} a n}$ is the category of strongly causal, $n$-dimensional spacetimes $(\widehat{\mathscr{M}}, \widehat{g})$, with arrows defined by orientation-preserving isometric embeddings with open, causally convex images, and $\mathscr{A} l g$ the category of unital $\mathrm{C}^{*}$ algebras, whose arrows are unit-preserving $\mathrm{C}^{*}$-morphisms. A locally covariant quantum theory is simply a covariant functor $\mathfrak{A}$ between both categories, i.e., the diagram

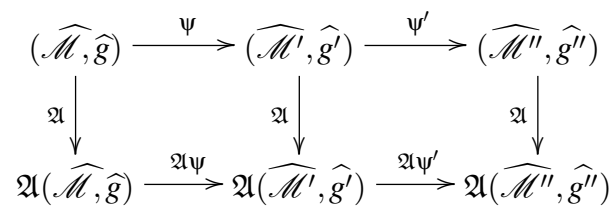

commutes. We say that $\mathfrak{A}$ is causal if the local algebras at spacelike separated regions commute, and primitively causal if the embedding of any neighborhood of a Cauchy surface into its Cauchy development induces an isomorphism of the respective algebras. In the latter case, one can define retarded and advanced "scattering morphisms" by suitable metric perturbations. The composition of both gives an automorphism $\beta_{\widehat{g}}$ of $\mathfrak{A}(\widehat{\mathscr{M}}, \widehat{g})$ (relative Cauchy evolution) whose functional derivative

$$
\frac{i}{2}\left\langle\Phi,\left[T^{\mu v}(x), \pi_{\omega}(A)\right] \Phi\right\rangle \doteq \frac{\delta}{\delta \widehat{g}_{\mu v}(x)}\left\langle\Phi, \pi_{\omega}\left(\beta_{\widehat{g}}(A)\right) \Phi\right\rangle
$$

acts in the same way (i.e., as a densely defined derivation) as the commutator with the energy-momentum tensor, in the sense of quadratic forms, on the GNS Hilbert space induced by a state satisfying the microlocal spectrum condition, endowed with boundary conditions in the following sense: it approaches an "Rindler-Unruh" type equilibrium state after a sufficiently long relaxation time - for such states, it's meaningful to speak about the implementation of asymptotic isometries. This derivation on the local algebras shares many properties of the Peierls bracket, and the splitting of the second variation into their retarded and advanced parts is also subject to renormalization ambiguities, in the same sense as above.

\section{Acknowledgments}

I thank Prof. Michael Forger for enlightening discussions on the principle of locality in classical field theory, which led to the crucial physical insights at the basis of the present work. This work is supported by FAPESP (grant no. 01/14360-1).
[1] I. Papadimitriou, K. Skenderis, arXiv:hep-th/0505190, and references therein.

[2] J. Maldacena, Adv. Theor. Math. Phys. 2, 231 (1998).

[3] E. Witten, Adv. Theor. Math. Phys. 2, 253 (1998).

[4] G. Scharf, Finite Quantum Electrodynamics - the Causal Approach. 2nd. ed., Springer-Verlag, 1995.

[5] S. Hollands, A. Ishibashi, and D. Marolf, arXiv:hep-th/0503105, and references therein.
[6] R. Brunetti, K. Fredenhagen, and R. Verch, Commun. Math. Phys. 193, 449 (1998).

[7] H. Friedrich, J. Geom. Phys. 17, 125 (1995).

[8] P. L. Ribeiro, arXiv:hep-th/0502096.

[9] R. Haag. Local Quantum Physics. 2nd. ed., Springer-Verlag, 1996. 